\title{
Design, Optimization, and Evaluation of a Novel Metronidazole-Loaded Gastro-Retentive pH-Sensitive Hydrogel
}

\author{
Galal M. El-Mahrouk, ${ }^{1}$ Mona H. Aboul-Einien, ${ }^{1,2}$ and Amal I. Makhlouf ${ }^{1}$
}

Received 24 August 2015; accepted 3 December 2015; published online 21 December 2015

\begin{abstract}
Floating $\mathrm{pH}$-sensitive chitosan hydrogels containing metronidazole were developed for the eradication of Helicobacter pylori from the stomach. Hydrogels were prepared by crosslinking medium or high molecular weight chitosan in lyophilized solutions containing metronidazole using either citrate or tripolyphosphate (TPP) salts at $1 \%$ or $2 \%$ concentration. A $2^{3}$ factorial design was developed to study the influence of formulation parameters on the physical characteristics of the prepared hydrogels. The interaction between hydrogel components was investigated. The morphology of the prepared hydrogels was inspected and their percentage swelling, release pattern, and moisture content were evaluated. The results revealed the absence of interaction between hydrogel components and their highly porous structure. Percentage swelling of the hydrogels was much higher, and drug release was faster in gastric $\mathrm{pH}$ compared with intestinal $\mathrm{pH}$. The formula prepared using $2 \%$ high molecular weight chitosan and $2 \%$ TPP significantly swelled (700\%) within the first $4 \mathrm{~h}$ and released the loaded drug over a period of $24 \mathrm{~h}$. Its moisture content was not affected by storage at high relative humidity. Therefore, this formula was selected to be tested in dogs for its gastric retention (using X-ray radiography) and efficacy in the eradication of $H$. pylori (using histopathological and microbiological examination). The results revealed that the prepared hydrogel formula was retained in dog stomach for at least $48 \mathrm{~h}$, and it was more effective against $H$. pylori than the commercially available oral metronidazole tablets (Flagyl@).
\end{abstract}

KEY WORDS: chitosan; floating drug delivery system; gastric retention; metronidazole; $\mathrm{pH}$-sensitive swelling.

\section{INTRODUCTION}

Helicobacter pylori was confirmed as the main pathogenic cause of peptic ulcer (1). Accordingly, several attempts have been made by researchers for efficient eradication of such micro-organism from the stomach. Metronidazole is an active adjunct in treatment of $H$. pylori by interference with DNA of the bacterium (2). The commonly reported side effects of metronidazole include anorexia, nausea, vomiting, epigastric pain, and antibiotic-associated colitis in addition to adverse effects on the nervous system. As the side effects of metronidazole are dose dependent and increase by prolonged therapy (3), it would be beneficial to formulate it in site-specific dosage forms, which act locally at the site of infection. This would reduce the therapeutic dose of the drug, minimize the possible side effects, and increase the efficiency of the treatment. Given that the H. pylori lives deep in the gastric mucosa, a logical way to improve the effectiveness of its treatment is to develop gastro-retentive dosage forms in order to release

\footnotetext{
${ }^{1}$ Department of Pharmaceutics and Industrial Pharmacy, Faculty of Pharmacy, Cairo University, Kasr El-Eini St., 11562, Cairo, Egypt.

${ }^{2}$ To whom correspondence should be addressed. (e-mail: mona.abouleinien@pharma.cu.edu.eg)
}

drugs as long as possible in the near vicinity of the bacterium (4). Floating drug delivery systems remain buoyant in the stomach without being affected by the gastric emptying for a prolonged period. While the system is floating on the gastric contents, the drug is slowly released at a pre-determined rate (5).

Hydrogels are of special interest in controlled release applications because of their soft tissue biocompatibility, the ease of which drugs are dispersed in their matrix, the high degree of controlling drug release, and the high drug-loading capacity compared with other delivery systems (6). In 2007, Ishak et al. developed floating alginate beads loaded with metronidazole. The floating beads proved much efficiency against $H$. pylori compared with metronidazole suspension (7).

"Intelligent" or "smart" hydrogels can control drug release by changing the gel structure in response to environmental stimuli such as $\mathrm{pH}$, electric fields, temperature, light, pressure, sound, and magnetic fields (8). Rajinikanth et al. (9) formulated Gellan-based amoxicillin floating in situ gelling system, which was more effective than oral amoxicillin suspension in the eradication of $H$. pylori in Mongolian gerbils. Chitosan is one of the most known safe polymers in smart hydrogel preparation because it is biocompatible, biodegradable, and bacteriostatic (10). Also, it promotes wound healing $(11,12)$ and acts as a penetration enhancer (13). Like other smart hydrogels, chitosan hydrogels are either covalently or 
ionically crosslinked. Covalently crosslinked chitosan hydrogels do not exhibit $\mathrm{pH}$-sensitive swelling or drug release behavior because of the strong covalent bonds between chitosan chains while ionically crosslinked chitosan networks generally exhibit $\mathrm{pH}$-sensitive swelling and drug release through their porous structure as proved by Bergera et al. (14).

This paper aimed to design and optimize chitosan $\mathrm{pH}-$ sensitive floating hydrogel loaded with metronidazole for the eradication of gastric $H$. pylori. A $2^{3}$ factorial design was developed to study the effect of formulation parameters such as chitosan molecular weight, crosslinker type, and concentration on the physicochemical characteristics of the prepared hydrogels. The optimized hydrogel formula was tested in dogs for its buoyancy and efficiency in the eradication of $H$. pylori compared with oral commercially available metronidazole tablets, Flagyl®.

\section{MATERIALS AND METHODS}

\section{Materials}

Metronidazole was provided by Farchemia, Italy. High molecular weight chitosan $(600,000 \mathrm{Da}$, viscosity of $1 \%$ solution in $1 \%$ acetic acid is $400 \mathrm{cps}$, degree of deacetylation 75$80 \%$ ) was purchased from Fluka Biochemica (Japan). Medium molecular weight chitosan (400,000 Da, viscosity of $1 \%$ solution in $1 \%$ acetic acid is $200 \mathrm{cps}$, degree of deacetylation $75-80 \%$ ) and pentasodium triphosphate (TPP) were obtained from Sigma Aldrich Chemie (products of Iceland and Germany, respectively). Karl-Fischer reagent was purchased from Riedel-Dehaën (Germany). H. pylori selective supplement (Dent's), gas generating kit, Campylobacter system BR0056A, and Columbia agar were supplied by Oxoid Ltd. (England). All other chemicals were of analytical grade and used as received. All water used was distilled de-ionized water.

\section{Preparation of Chitosan Hydrogels}

Hydrogels were prepared using $4 \%$ high or medium molecular weight chitosan solution in $2 \%$ acetic acid in which metronidazole was homogenously dispersed. A specified volume of the dispersion $(5 \mathrm{ml}$ ) containing $250 \mathrm{mg}$ metronidazole was frozen at $-4^{\circ} \mathrm{C}$ overnight and freeze dried for $24 \mathrm{~h}$ at a condenser temperature of $-50^{\circ} \mathrm{C}$ and a pressure of $7 \times 10^{-2}$ mbar. (Lyophilizer, Novalyphe N.L.500, Savant Instrument, Halbrook, NY, USA). The lyophilized product was sunk in the appropriate electrolyte solution (either TPP or sodium citrate at $2 \%$ or $1 \%$ concentration) for 30 min then wrapped in aluminum foil and dried overnight in an oven at $50^{\circ} \mathrm{C}$. The resultant $2^{3}$ factorial design is given in Table I.

\section{Interaction Between pH-Sensitive Chitosan Hydrogel Components}

Differential scanning calorimetry (DSC) thermograms, Fourier transform-infrared (FTIR) spectra, and X-ray diffractograms were recorded for metronidazole powder, medium molecular weight chitosan, drug-polymer physical mixture (1:1), and the prepared chitosan hydrogels (formulas F1-F8).
Table I. Composition of the Hydrogel Formulas Prepared According to $2^{3}$ Factorial Design

\begin{tabular}{lllc}
\hline Formula & $\begin{array}{l}\text { Chitosan molecular } \\
\text { weight }\end{array}$ & $\begin{array}{l}\text { Crosslinker } \\
\text { type }\end{array}$ & $\begin{array}{l}\text { Crosslinker } \\
\text { concentration }\end{array}$ \\
\hline F1 & High & TPP $^{\mathrm{a}}$ & $2 \%$ \\
F2 & High & TPP $^{\mathrm{a}}$ & $1 \%$ \\
F3 & High & Sodium citrate & $2 \%$ \\
F4 & High & Sodium citrate & $1 \%$ \\
F5 & Medium & TPP $^{\mathrm{a}}$ & $2 \%$ \\
F6 & Medium & TPP $^{\mathrm{a}}$ & $1 \%$ \\
F7 & Medium & Sodium citrate & $2 \%$ \\
F8 & Medium & Sodium citrate & $1 \%$ \\
\hline
\end{tabular}

${ }^{a}$ TPP pentasodium triphosphate

Composition of the formulas is given in Table I

\section{Differential Scanning Calorimetry Study}

DSC analysis was performed using a Shimadzu differential scanning calorimeter (DSC-50, Shimadzu, Japan). The apparatus was calibrated with purified indium (99.9\%). Samples (3-4 mg) were placed in flat-bottomed aluminum pan and heated at a constant rate of $10^{\circ} \mathrm{C} / \mathrm{min}$, in an atmosphere of nitrogen in a temperature range of $20-400^{\circ} \mathrm{C}$.

\section{Fourier Transform-Infrared Spectroscopy Study}

The FTIR spectra were recorded using a Bruker FTIR spectrophotometer (Model 22, Bruker, UK) according to the $\mathrm{KBr}$ disc technique. Smoothing of the spectra and baseline correlation procedures were applied. The spectra were saved using a Lotus123 computer program. The FTIR measurements were performed in the scanning range of 4000 $400 \mathrm{~cm}^{-1}$ at ambient temperature.

\section{$X$-ray Diffractometry Study}

The X-ray diffraction patterns were recorded at room temperature using a Scintag diffractometer (XGEN-4000, Scintag Corp., USA). The samples were irradiated with Nifiltered $\mathrm{Cu}$ Ka radiation, at $45 \mathrm{kV}$ voltage and $40 \mathrm{~mA}$ current. The scanning rate employed was $2 \%$ min over a diffraction angle of $2 \theta$ and range of $3-70^{\circ}$.

\section{Physicochemical Evaluation of the Prepared Chitosan Hydrogels}

\section{Weight Uniformity}

Six units of each of the prepared formulas were weighed separately. The mean weight was calculated for each formula along with the standard deviation.

\section{Drug Content Uniformity}

The drug content was determined for each formula by stirring the prepared hydrogel in $1000 \mathrm{ml} 0.1 \mathrm{~N} \mathrm{HCl}$ for $48 \mathrm{~h}$, then measuring the UV absorbance of metronidazole in the solution at a wave length $=277 \mathrm{~nm}$ (Spectrophotometer; UV- 
1601, Shimadzu, Japan). For each of the prepared hydrogel formulas, the mean drug content in six units as well as the standard deviation was calculated.

\section{Residual Moisture Content}

The prepared chitosan hydrogel formulas were analyzed for their residual moisture content using a Karl Fischer titrator (Veego, Matic-MD, Veego Instruments Corporation, India). A known weight of the sliced hydrogel was inserted in the titration vessel containing dried methanol (Karl-Fisher grade) and titrated with Hydranal Composite 5 reagent after a stirring time of $2 \mathrm{~min}$. Results are presented as mean values of three samples $\pm \mathrm{SD}$.

\section{Swelling Measurement}

The swelling of the prepared hydrogel formulas was studied in simulated gastric fluid (SGF) as well as in Sorensen's phosphate buffer of $\mathrm{pH}$ 7.4. The USP Dissolution Tester Apparatus II U.S.P (Pharma Test, Germany) was used, where the pre-weighed hydrogel was immersed in $900 \mathrm{ml}$ of the medium, the paddle speed was set at $50 \mathrm{rpm}$, and the temperature was maintained at $37 \pm 0.5^{\circ} \mathrm{C}$. At different time intervals, the hydrogel was removed, excess water was plotted from its surface, and it was weighed. The following equation was used to calculate percentage swelling of hydrogels (15):

$\%$ swelling $=\left(W_{t}-W_{0} / W_{0}\right) \times 100$

Where, $W_{t}$ and $W_{0}$ are the sample weights at time $t$ and in the dry state, respectively. All experiments were done in triplicates, and the results were expressed as mean values $\pm \mathrm{SD}$.

\section{Buoyancy of the Prepared Hydrogels}

During the swelling measurement experiment, the ability of the prepared hydrogels to float was noticed. The time period during which the hydrogel was able to float was recorded for each formula in both SGF ( $\mathrm{pH} 1.2)$ and in Sorensen's phosphate buffer of $\mathrm{pH}$ 7.4.

\section{IN VITRO RELEASE STUDY}

The release of metronidazole from the prepared hydrogels (of $250 \mathrm{mg}$ unit dose) was studied in both SGF and Sorensen's phosphate buffer of $\mathrm{pH}$ 7.4. The same conditions described previously in the swelling measurement experiment were used for release studies. Samples were taken from the release medium at predetermined time intervals, and they were spectrophotometrically analyzed for their metronidazole content after appropriate dilution. The wavelength used was $277 \mathrm{~nm}$ for samples of SGF and $320 \mathrm{~nm}$ for those of phosphate buffer ( $\mathrm{pH}$ 7.4). The experiment was repeated three times for each formula and the mean values as well as standard deviation were calculated.

\section{Characterization of Hydrogel Formula F1}

\section{Scanning Electron Microscopy}

The surface morphology of the prepared hydrogel formula F1 was examined using scanning electron microscope (microanalyzer, Jeol, JXA-840 A, Japan). Samples were mounted on metal grids using double- sided adhesive tape. They were coated with gold under argon atmosphere prior to observations.

\section{Moisture Uptake on Storage}

The prepared hydrogel formula $\mathrm{F} 1$ was stored in desiccators at room temperature $\left(15-30^{\circ} \mathrm{C}\right)$ and relative humidity of $75 \%$ for 6 months. The moisture content of the formula was estimated at the beginning of the experiment and after 1, 2, 4, and 6 months. The same method described for measurement of the moisture content of freshly prepared hydrogels was applied. All measurements were carried out in triplicates, and the mean values \pm SD were calculated.

\section{STATISTICAL ANALYSIS OF DATA}

Factorial analysis including the analysis of variance (ANOVA) test and the subsequent Fisher pairwise least significant difference test (PLSD) test was performed using the statistical software Statview for Windows ${ }^{\circledR}$ (Abacus Concept Inc., version 4.57, 1992-1996).

\section{IN VIVO EVALUATION OF THE PREPARED HYDROGEL FORMULA F1 IN DOGS}

\section{Preparation of Radio-opaque Metronidazole Floating Hydrogel Formula F1}

The floating hydrogel formula F1 was prepared as mentioned previously and made radio-opaque by the addition of $1 \mathrm{ml} 50 \%$ barium sulfate suspension, which was homogenously mixed with the dispersion before being frozen and lyophilized. The prepared radio-opaque hydrogel formula was then packed in size 000 capsules (Capsulin $®$, USA).

\section{Radiographic Examination of Formula F1 in Dogs}

Three dogs were used in this experiment. The dogs were fasted overnight, then fed with about $500 \mathrm{~g}$ canned food and $100 \mathrm{ml}$ water directly before administration of the capsule containing the radio-opaque hydrogel. Radiographs were taken from a lateral view at zero time, $15 \mathrm{~min}$, half an hour, and 2 , $4,8,24$, and $48 \mathrm{~h}$ after dosing. The X-ray generating unit (Fischer Imaging R183) was set at $50 \mathrm{kv}, 100 \mathrm{mAm}$, and $0.1 \mathrm{~s}$. The protocol of this experiment was approved by The University Protection of Experimental Animals Committee. 


\section{Efficacy of Formula F1 in the Treatment of $\boldsymbol{H}$. pylori-Infected Dogs}

\section{Preparation of Pathogenic H. pylori Culture}

The culture preparation was performed in the GIT Endoscope Unit at The National Hepatology and Tropical Medicine Research Institute, Cairo, Egypt. The study was approved by the University Protection of Human Subjects Committee, and the applied protocol complies with the declarations of Helsinki and Tokyo for humans. Gastric biopsy specimens were taken from patients $(n=10)$ with gastritis, gastric, and duodenal ulcer through the gastroduodenoscope. Each taken biopsy was homogenized with sterile saline $(0.5 \mathrm{ml} / \mathrm{stomach})$ and plated on Columbia agar plates containing $5 \%$ horse blood supplemented with $H$. pylori selective supplement (Dent's) antibiotic mixture. The plates were incubated for 5 days at $37^{\circ} \mathrm{C}$ under microaerophilic conditions, using gas generating kit in GasPak® jar. Growing H. pylori colonies were identified by their typical morphology, as assessed by visual inspection of the plates, and confirmed by urease and oxidase tests as described by Rossi et al. (16).

\section{Animals and Experimental Design}

The protocol of animal experiments was approved by The University Protection of Experimental Animals Committee and complies with The European Community Guidelines for the use of experimental animals. Ten breed dogs weighing about $15 \mathrm{~kg}$ each were used. The dogs were housed in individual cages and maintained on standard diet of dry food and ad libitum access to water. The experiment was divided into four stages:

Stage I Preparation of dogs:

This stage lasted for 2 weeks, during which all dogs were given a combination of amoxicillin (20 mg/kg), metronidazole (30 mg/kg), and cimetidine $(20 \mathrm{mg} / \mathrm{kg})$ once daily. At the end of stage I, one dog was euthanized, by an overdose of intravenous thiopental ( $0.5 \mathrm{~g}$ USPXXI for intravenous anaesthesia, EPICO, Egypt), and its stomach was removed, examined, and taken as a negative control.

Stage II Inoculation of $H$. pylori:

This stage lasted for 1 week, during which the remaining nine dogs were challenged every other day, for a total of four times, with $3 \mathrm{ml}$ of a freshly prepared suspension of $H$. pylori in sterile saline containing $10^{9}$ colony forming unit (CFU) (16). The dogs were fasted for $24 \mathrm{~h}$ before each challenge with ad libitum access to water.

Stage III Colonization and pathogenesis of H. pylori: This stage lasted for 4 weeks. Dogs were maintained on a diet of dry food and tap water to allow for the colonization and the pathogenesis of the inoculated organisms to take place. At the end of the fourth week, three dogs were euthanized and their stomachs were removed, examined, and taken as a positive control group (group A) to ascertain the inoculation of $H$. pylori in the stomach of dogs.
Stage IV Treatment:

This stage lasted for 2 weeks. The six remaining dogs were divided into two groups, B and C, each containing three animals. Group B dogs received Flagyl ${ }^{\circledR}$ tablets $(250 \mathrm{mg}$ metronidazole, Aventis, Egypt), and group $\mathrm{C}$ animals received the selected hydrogel formula F1 (250 mg metronidazole, packed in size 000 capsules) once daily. The dogs were fasted overnight before dosing. Each dog was fed with about $500 \mathrm{~g}$ canned food and $100 \mathrm{ml}$ water directly before dosing. One day after administration of the last dose, the dogs were euthanized and their stomachs were removed and examined.

\section{Examination of the Removed Stomachs}

The removed stomach from the negative control dog was subjected to macroscopic examination of gastric mucosa to assure normal morphology. In addition, biopsies were taken from different sites in the stomach for urease test and histopathological examination. Three samples were taken from the antrum, three from the fundus, three from the cardia, and one biopsy from the pylorus. The taken samples were subjected to urease test according to the method described by Rossi et al. (16). Biopsies for histopathological examination were fixed in $15 \%$ formalin for 3 days and embedded in paraffin. Four to five-micrometer-thick sections were cut by microtome and stained with hematoxylin-eosin (HE).

The removed stomachs from dogs of groups A, B, and C were subjected to macroscopic examination of gastric mucosa to detect any abnormality such as lesions, ulcers, or congestion. Histopathological examination was carried out as for the negative control stomach. In addition, samples for microbial count were taken from the same sites as for histopathological examination. For microbial count, samples from each dog were separately homogenized with $3 \mathrm{ml}$ normal saline from which serial dilutions were made and cultured under the same conditions as for the preparation of pathogenic H. pylori culture described above. Viable cell count (expressed as $\mathrm{CFU} / \mathrm{ml}$ ) was calculated for each dog by counting the number of colonies on the agar plates. The colonies were identified as H. pylori by morphology, oxidase, and urease activity (16).

\section{Statistical Analysis}

Student $t$ test was performed to test the significance of the difference in colony count between groups $\mathrm{A}, \mathrm{B}$, and $\mathrm{C}$.

\section{RESULTS}

\section{Interaction Between pH-Sensitive Chitosan Hydrogel Components}

\section{Differential Scanning Calorimetry Study}

The DSC thermograms of metronidazole, medium molecular weight chitosan, drug/polymer physical mixture (1:1), and the prepared formulas (F1-F8) are illustrated in Fig. 1. The DSC thermograms of metronidazole/chitosan physical mixture and the prepared hydrogels revealed that the endothermic peak of the drug was reserved, but it became slightly shorter. 


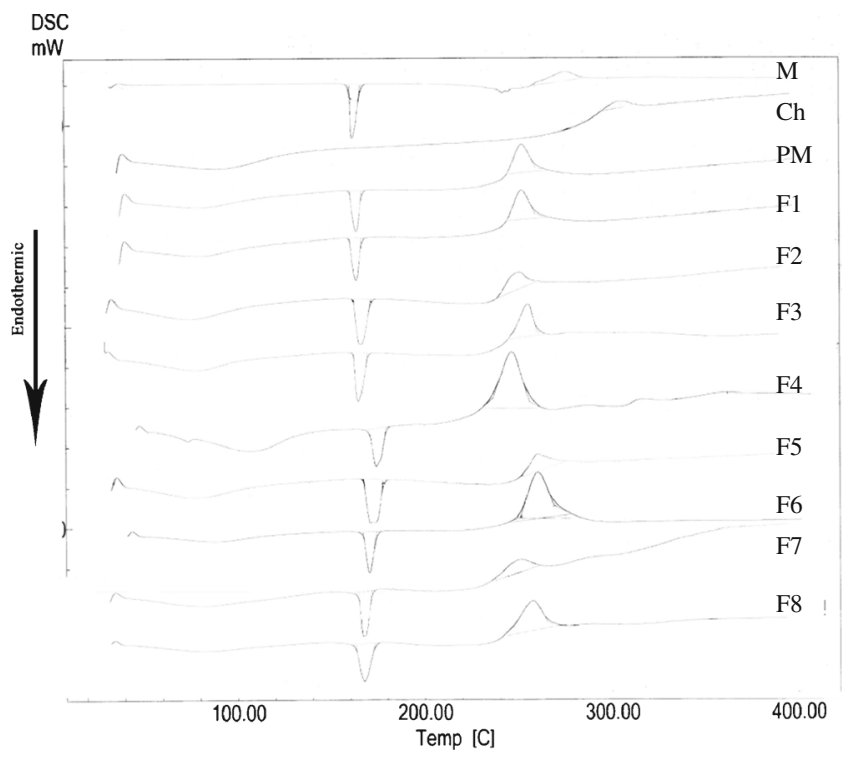

Fig. 1. DSC thermograms of metronidazole $(M)$, chitosan $(C h)$, metronidazole/chitosan (1:1) physical mixture $(P M)$, and the prepared chitosan hydrogel formulas: $F 1-F 8$. Composition of the formulas is given in Table I

\section{Fourier Transform-Infrared Spectroscopy Study}

The infrared spectra of the tested samples are given in Fig. 2. As shown in Fig. 2, the infrared spectra of the prepared formulas (F1-F8) exhibited the characteristic bands of metronidazole. In addition, the infrared spectrum of metronidazole/ chitosan physical mixture was nearly superimposed with that of the prepared hydrogel formulas.

\section{$X$-ray Diffractometry Study}

The X-ray diffractograms of the tested samples are shown in Fig. 3. Figure 3 reveals that the intensity of the characteristic peaks of metronidazole decreased in the difractogram of drug-chitosan physical mixture as well as in those of the prepared hydrogels.

\section{Physicochemical Evaluation of the Prepared Chitosan Hydrogels}

\section{Weight Uniformity}

The mean values of formula weight are represented in Table II. One-way ANOVA test on these results produced an $F$ value $=2.3201$ and $p$ value $>0.05$.

\section{Drug Content Uniformity}

The mean drug content of each of the prepared hydrogel formulas along with its SD are shown in Table II. The applied one-way ANOVA test calculated an $F$ value of 0.0781 and a $p$ value exceeding 0.05. As shown in Table II, the drug content in the prepared formulas ranged from $246 \pm 4.24$ (for formula F2) to $249.5 \pm 10.60$ (for formula F7); representing 98.4 $\pm 1.70 \%$ and $99.8 \pm 4.24 \%$ of the initially added drug amount, respectively.

\section{Residual Moisture Content}

The calculated values of residual moisture content of each of the prepared chitosan hydrogels are given in Table II. As shown in Table II, the residual moisture did not exceed $5.97 \pm 0.59 \%$ of the formula weight for any of the prepared formulas. One-way ANOVA test on the results calculated an $F$ value $=0.94$ and $p$ value $>0.05$.

\section{Swelling Measurement}

Table III summarizes the swelling ability of the prepared hydrogels in $\mathrm{pH}$ values 1.2 and 7.4 at $37^{\circ} \mathrm{C}$. All the prepared formulas showed remarkable swelling in SGF ( $\mathrm{pH} 1.2$ ), ranging from $183 \pm 76 \%$ to $762 \pm 31 \%$, within short times $(0.5-8 \mathrm{~h})$ as revealed by Table III. However, the percentage swelling of the same formulas in phosphate buffer $(\mathrm{pH}$ 7.4) did not exceed $177 \pm 7 \%$ within $24 \mathrm{~h}$.

Results in Table III also show that in SGF ( $\mathrm{pH}$ 1.2), the swelling ability of formulas prepared using sodium citrate as crosslinker was less than that for the corresponding formulas prepared using TPP. The percentage swelling of the former formulas (F3, F4, F7, and F8) did not exceed $328 \pm 20 \%$, while all of the latter ones (F1, F2, F5, and F6) showed more than $490 \pm 25 \%$ swelling.

During swelling measurements in SGF, only formula F1 retained its physical shape for the 24 -h period of the experiment. Other formulas dissociated gradually after swelling. The dissociation was faster for formulas prepared using medium (rather than high) molecular weight chitosan and lower concentration of the crosslinking agent.

\section{Buoyancy of the Prepared Hydrogels}

All the prepared formulas remained floating for $24 \mathrm{~h}$ in both SGF and Sorensen's phosphate buffer of pH 7.4 even during their dissociation.

\section{In Vitro Release Study}

The release profiles of metronidazole from the prepared chitosan hydrogels (formulas F1-F8) in SGF and Sorensen's phosphate buffer of $\mathrm{pH} 7.4$ are presented in Figs. 4 and 5. These figures show that for all the prepared formulas, more than $70 \%$ of the loaded drug was released in SGF ( $\mathrm{pH} 1.2)$ within $24 \mathrm{~h}$, with a percent drug release approaching $90 \%$ for some formulas. However, in phosphate buffer ( $\mathrm{pH} 7.4)$, none of the prepared formulas released more than $70 \%$ of the loaded drug within the $24 \mathrm{~h}$ of the experiment. For reasons of comparison, the time required for $50 \%$ of the loaded drug to be released $\left(t_{50 \%}\right)$ was estimated for each formula, and the results are represented in Table IV. Results of $t_{50 \%}$ were subjected to statistical analysis using two-way ANOVA test and Fisher's PLSD.

The $F$ values were calculated for the three studied formulation factors namely, the molecular weight of the used chitosan, the type of the used crosslinking agent, and its concentration. The calculated $F$ values for the studied formulation factors were $1178.973,413.256$, and 16.11 , respectively with $p$ values $\leq 0.0001$ for the first two factors and $<0.05$ for the third one.

The results of drug release from each hydrogel formula in SGF ( $\mathrm{pH}$ 1.2) were subjected to kinetic analysis to determine the release model. The regression coefficient $\left(R^{2}\right)$ of the best 

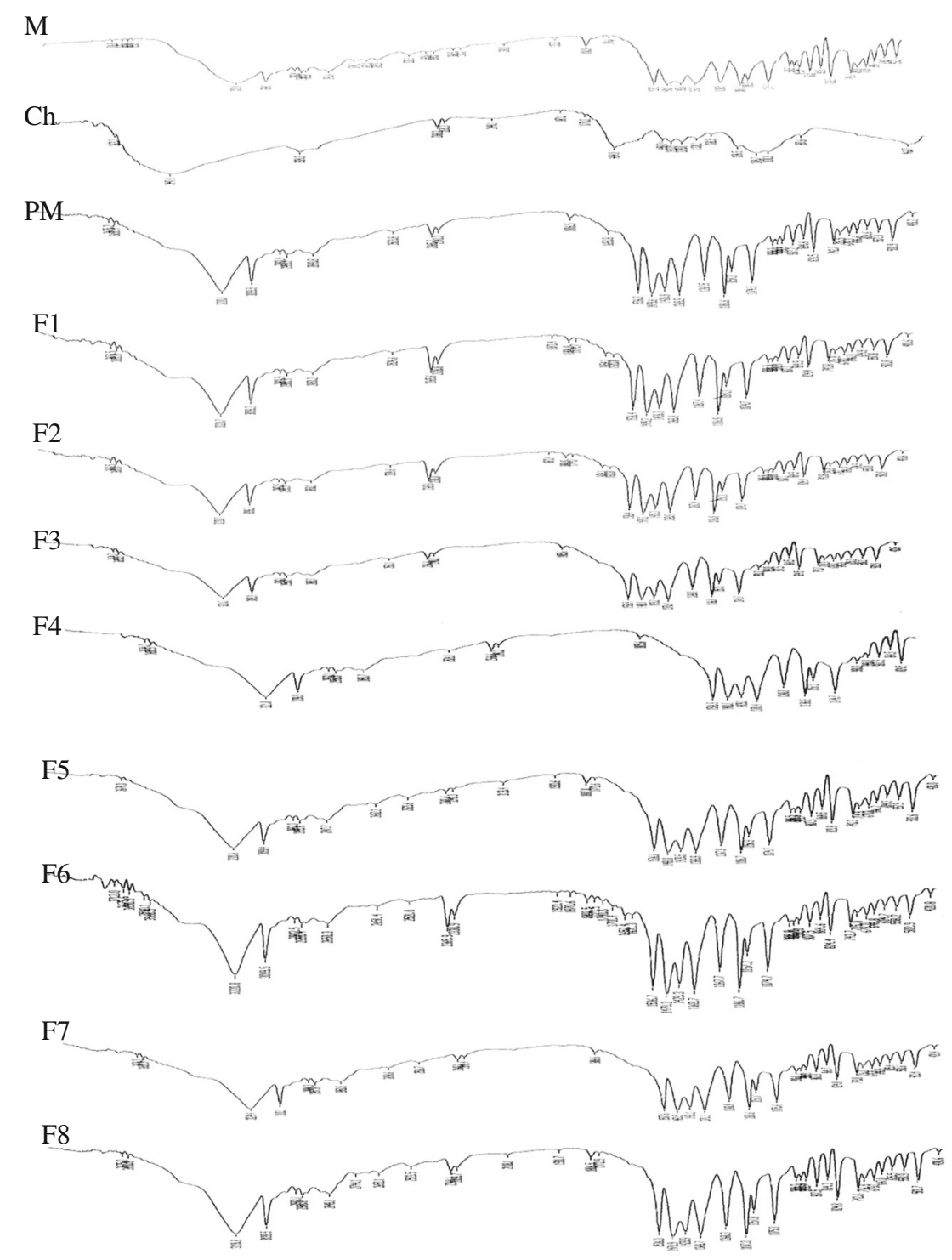

Fig. 2. Infrared spectra of metronidazole $(M)$, chitosan $(C h)$, metronidazole/chitosan (1:1) physical mixture $(P M)$, and the prepared chitosan hydrogel formulas: $F 1-F 8$. Composition of the formulas is given in Table I

statistical line of each model is presented in Table IV. As shown in Table IV, formulas F1, F2, F4, F6, and F7 followed first-order drug release kinetics while formulas F3 and F8 followed zero-order kinetics and formula F5 showed Higuchi diffusion kinetics.

Furthermore, the initial $60 \%$ drug release data were fitted to the following empirical formula of Ritger and Peppas (17):

$M_{t} / M_{\infty}=k_{t}^{n}$

Where, $M_{t} / M_{\infty}$ is the fraction of the drug released at time $t, k$ is the kinetic parameter, and $n$ is the diffusion exponent. By applying the least squares regression method to the plots of $\log M_{t} / M_{\infty}$ versus $\log t$ in the above equation, the values of $n$ were determined and are presented in Table IV. As shown in
Table IV, the values of $(n)$ ranged from 0.6 to 0.9 for all formulas.

\section{CHARACTERIZATION OF HYDROGEL FORMULA F1}

\section{Scanning Electron Microscopy}

The surface morphology of hydrogel formula F1 is presented in Fig. 6. The presented micrograph reveals the highly porous matrix with large, open, channel-like structure of the hydrogel.

\section{Moisture Uptake on Storage}

The results of the moisture content were estimated for formula F1 after 1, 2, 4, and 6 months (results not shown). 


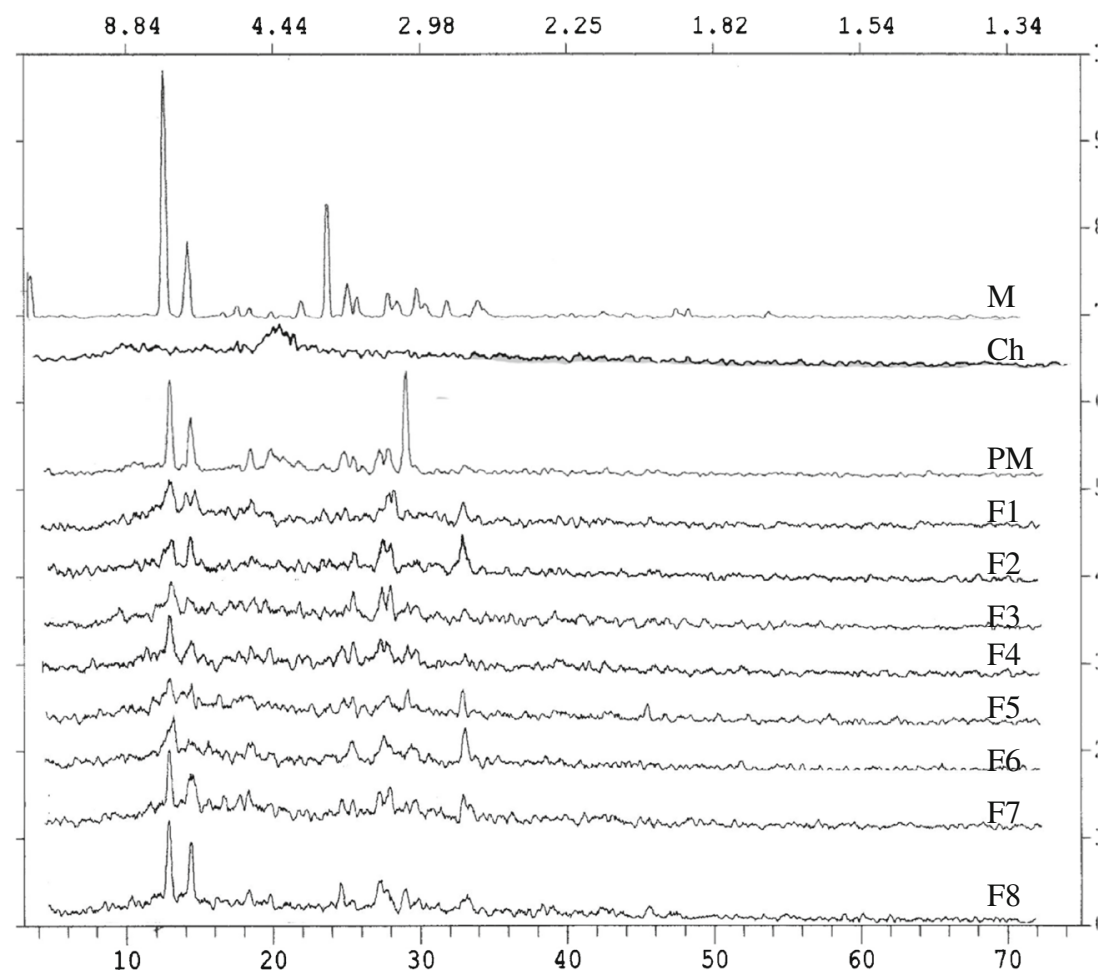

Fig. 3. X-ray diffractograms of metronidazole $(M)$, chitosan $(C h)$, metronidazole/chitosan (1:1) physical mixture $(P M)$, and the prepared chitosan hydrogel formulas: $F 1-F 8$. Composition of the formulas is given in Table I

These results were subjected to statistical analysis using oneway ANOVA test to find out the significance of differences from results obtained by freshly prepared formula. The calculated $p$ values were $>0.05$.

\section{IN VIVO EVALUATION OF THE PREPARED HYDROGEL FORMULA F1 IN DOGS}

\section{Radiographic Examination of Formula F1 in Dogs}

Figure 7 demonstrates the location of the hydrogel in dog's stomach over a period of $48 \mathrm{~h}$ after ingestion of the

Table II. Physicochemical Evaluation of the Prepared Hydrogels

\begin{tabular}{|c|c|c|c|}
\hline Formula $^{a}$ & $\begin{array}{l}\text { Formula weight }{ }^{b} \\
\quad(\mathrm{mg})\end{array}$ & $\begin{array}{l}\text { Drug content }{ }^{b} \\
\quad(\mathrm{mg})\end{array}$ & $\begin{array}{c}\text { Residual moisture } \\
\text { content }^{c}(\%)\end{array}$ \\
\hline $\mathrm{F} 1$ & $515 \pm 0.0071$ & $248.5 \pm 2.12$ & $5.12 \pm 0.74$ \\
\hline $\mathrm{F} 2$ & $495 \pm 0.0071$ & $246.0 \pm 4.24$ & $4.89 \pm 0.30$ \\
\hline $\mathrm{F} 3$ & $510 \pm 0.0141$ & $247.0 \pm 9.89$ & $4.95 \pm 0.50$ \\
\hline $\mathrm{F} 4$ & $505 \pm 0.0141$ & $246.0 \pm 5.65$ & $2.65 \pm 0.36$ \\
\hline F5 & $495 \pm 0.0071$ & $248.5 \pm 4.95$ & $5.74 \pm 0.83$ \\
\hline F6 & $485 \pm 0.0071$ & $249.0 \pm 8.48$ & $5.97 \pm 0.59$ \\
\hline F7 & $470 \pm 0.0071$ & $249.5 \pm 10.60$ & $6.24 \pm 0.97$ \\
\hline F8 & $490 \pm 0.0283$ & $247.5 \pm 2.121$ & $4.42 \pm 0.92$ \\
\hline
\end{tabular}

${ }^{a}$ Composition of the formulas is given in Table I

${ }^{b}$ Results are mean values $(n=6) \pm \mathrm{SD}$

${ }^{c}$ Results are mean values $(n=3) \pm \mathrm{SD}$ prepared radio-opaque metronidazole floating hydrogel formula F1. As revealed by Fig. 7, the swallowed hydrogel formula stayed in the stomach for $48 \mathrm{~h}$ during which it had altered its position and turned around. At $24 \mathrm{~h}$, the hydrogel began to take a higher anatomical position in the stomach; however, it could still alter its position and turn around in the gastric fluid. At $48 \mathrm{~h}$, the hydrogel remained in the stomach with further increase in size.

Table III. Swelling Measurements of the Prepared Hydrogels in Simulated Gastric Fluid (SGF; pH 1.2) and Phosphate Buffer (pH 7.4)

\begin{tabular}{|c|c|c|c|}
\hline \multirow[t]{2}{*}{ Formula $^{a}$} & \multicolumn{2}{|c|}{ Swelling profile in SGF } & \multirow{2}{*}{$\begin{array}{l}\% \text { swelling after } \\
24 \mathrm{~h} \text { in phosphate } \\
\text { buffer }^{b}\end{array}$} \\
\hline & $\begin{array}{l}\text { Maximum \% } \\
\text { swelling }^{b}\end{array}$ & $\begin{array}{l}\text { Time for } \\
\text { maximum } \\
\% \text { swelling (h) }\end{array}$ & \\
\hline $\mathrm{F} 1$ & $762 \pm 31$ & 24 & $177 \pm 7$ \\
\hline $\mathrm{F} 2$ & $699 \pm 20$ & 5 & $154 \pm 7$ \\
\hline F3 & $183 \pm 76$ & 4 & $155 \pm 4$ \\
\hline F4 & $223 \pm 24$ & 1 & $156 \pm 10$ \\
\hline F5 & $490 \pm 25$ & 8 & $168 \pm 9$ \\
\hline F6 & $566 \pm 182$ & 8 & $155 \pm 8$ \\
\hline F7 & $190 \pm 17$ & 0.5 & $162 \pm 10$ \\
\hline F8 & $328 \pm 20$ & 6 & $152 \pm 7$ \\
\hline
\end{tabular}


a In simulated gastric fluid ( $\mathrm{pH} 1.2)$

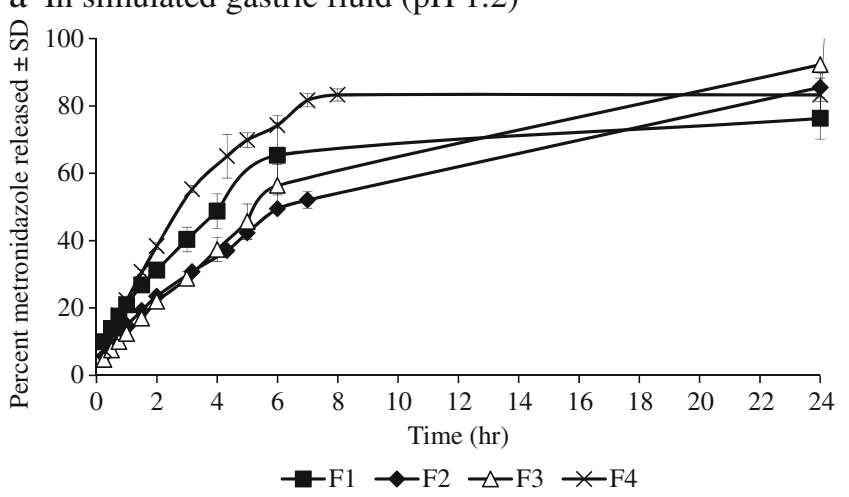

b In phosphate buffer ( $\mathrm{pH} 7.4)$

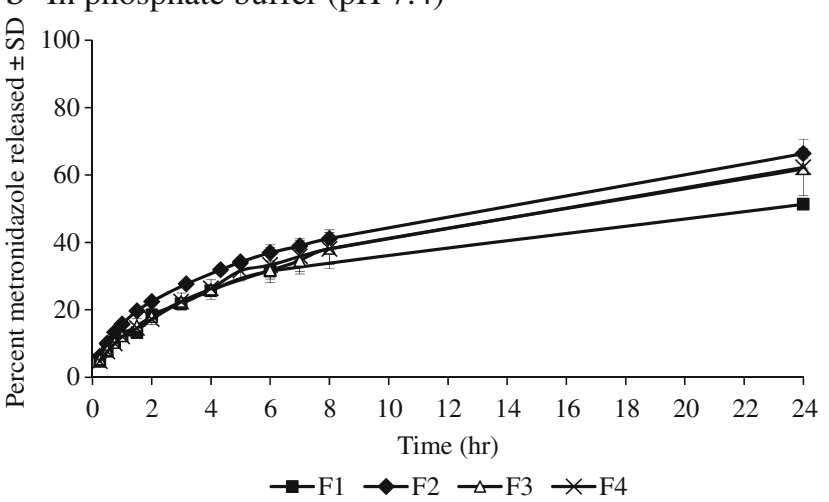

Fig. 4. Release profiles of metronidazole from the hydrogel formulas prepared using high molecular weight chitosan (F1-F4). Composition of the formulas is given in Table I

\section{Efficacy of Formula F1 in the Treatment of $H$. pylori-Infected Dogs}

The gastric biopsies taken from the stomach of the negative-control dog gave negative urease test. In addition, the macroscopic and histopathologic examination of these biopsies showed normal color and appearance as proven in Fig. 8a.

On the other hand, gastric biopsies taken from the stomach of the positive control dogs (group A) gave positive urease test. Macroscopically, the gastric mucosa in these biopsies appeared congested with few lesions. Histological examination of the gastric biopsies from group A dogs reveals the presence of hyperplasia of glandular epithelium, oedema in lamina propria, large numbers of inflammatory cells near the muscularis mucosa as well as vacuolation of the glandular epithelium of mucosa as shown in Fig. 8, parts b1-b4, respectively.

Macroscopic examination showed that, the gastric mucosa of animals in groups $\mathrm{B}$ and $\mathrm{C}$ was less congested than that of untreated (positive control) animals (group A). However, the gastric mucosa of group B dogs (treated with Flagyl ${ }$ ), was slightly more congested compared with that of group $\mathrm{C}$ animals (treated with formula F1). Histopathological examination of gastric mucosa of dogs in groups B and C is illustrated in Fig. 9. The histopathology of a group B dog stomach, shown in Fig. 9, revealed local areas of desquamation of epithelial lining mucosa accompanied by congestion of blood vessels in a In simulated gastric fluid ( $\mathrm{pH} 1.2)$

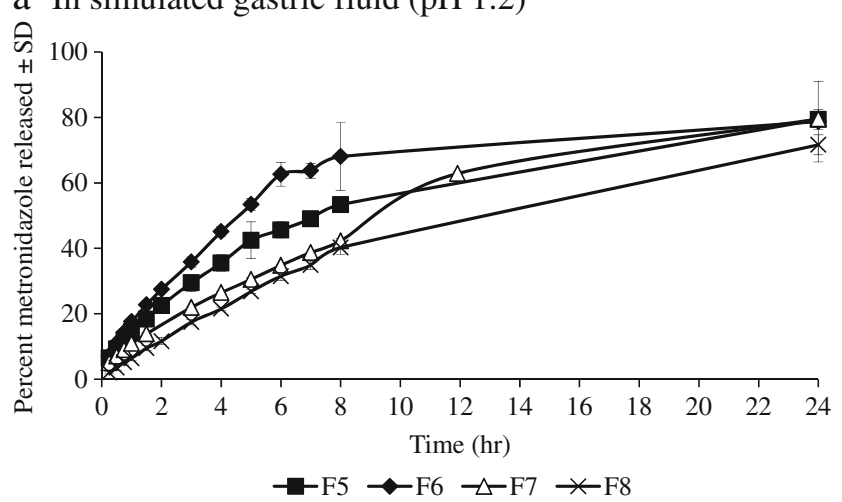

b In phosphate buffer ( $\mathrm{pH} 7.4)$

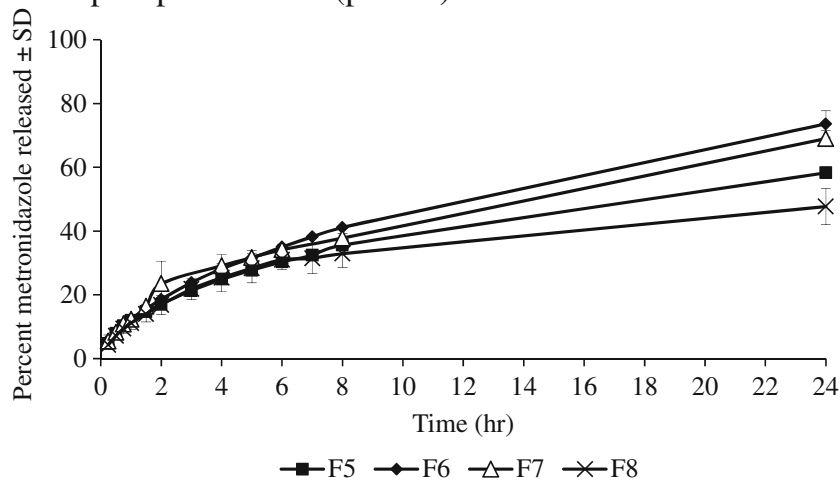

Fig. 5. Release profiles of metronidazole from the hydrogel formulas prepared using medium molecular weight chitosan (F5-F8). Composition of the formulas is given in Table I

both lamina propria and submucosa (Fig. 9(a1)). In addition, inflammatory cell infiltrations were observed in lamina propria either subepithelial or deeply near the muscularis mucosa (Fig. 9(a2)). On the other hand, the histopathology of a group C dog stomach, shown in Fig. 9(b1, b2), exhibited only mild congestion in lamina propria and submucosa with fewer numbers of inflammatory cells.

Independent sample $t$ test for data of the colony count revealed that both groups $\mathrm{B}$ and $\mathrm{C}$ animals showed significant reduction in colony count compared with group $\mathrm{A}$ ones. The calculated $t$ values were 2.8016 and 5.0990 for groups B and C, respectively, and the $p$ values did not exceed 0.05 for either group. Furthermore, group $\mathrm{C}$ dogs showed a statistically significant decrease in colony count compared with group B ones $(t=3.1892, p<0.05)$.

\section{DISCUSSION}

The endothermic peak of metronidazole was reserved in the DSC thermogram of drug-chitosan physical mixture (1:1), indicating the absence of physical interaction between the drug and the used polymer. The appearance of the characteristic bands of the drug in the infrared spectra of the prepared hydrogel formulas indicates the presence of metronidazole suspended in the hydrogel matrices. In addition, the infrared spectra of the drug in the prepared hydrogels and in its mixture with chitosan are nearly congruent signifying that there is 
Table IV. Metronidazole In Vitro Release Study from the Prepared Hydrogels

\begin{tabular}{|c|c|c|c|c|c|}
\hline \multirow[t]{2}{*}{ Formula $^{a}$} & \multirow[t]{2}{*}{$t_{50 \%}(\mathrm{~h})^{b}$} & \multicolumn{3}{|c|}{ Regression coefficient $\left(R^{2}\right)$} & \multirow[t]{2}{*}{ Release exponent $(n)^{d}$} \\
\hline & & Zero order & First order & Higushi & \\
\hline $\mathrm{F} 1$ & $4.167 \pm 0.59$ & 0.9881 & $0.9985^{c}$ & 0.9956 & 0.5947 \\
\hline $\mathrm{F} 2$ & $6.025 \pm 0.035$ & 0.9860 & $0.9955^{c}$ & 0.9939 & 0.6627 \\
\hline F3 & $5.39 \pm 0.0825$ & $0.9980^{c}$ & 0.9836 & 0.9720 & 0.7310 \\
\hline $\mathrm{F} 4$ & $2.68 \pm 0.023$ & 0.9940 & $0.9978^{c}$ & 0.9900 & 0.7720 \\
\hline F5 & $7.17 \pm 0.24$ & 0.9773 & 0.9946 & $0.9964^{c}$ & 0.6263 \\
\hline F6 & $4.5 \pm 0.24$ & 0.9935 & $0.9968^{c}$ & 0.9822 & 0.7801 \\
\hline F7 & $11.38 \pm 0.06$ & 0.9911 & $0.9987^{c}$ & 0.9943 & 0.6300 \\
\hline F8 & $12.83 \pm 0.25$ & $0.9988^{c}$ & 0.9986 & 0.9779 & 0.8693 \\
\hline
\end{tabular}

${ }^{a}$ Composition of the formulas is given in Table I

${ }^{b}$ Time required for $50 \%$ of the loaded drug to be released. Results are mean values $(n=3) \pm \mathrm{SD}$

${ }^{c}$ The highest values of $R^{2}$ represent the release model

${ }^{d}$ The release exponent $(n)$ was calculated according to Eq. 2

no chemical interaction between metronidazole and chitosan in the prepared formulas. The X-ray diffractogram of pure metronidazole proves its crystalline nature. However, the decrease in the intensity of the characteristic peaks of the drug in the diffractogram of drug-chitosan physical mixture indicates the decrease in drug purity by virtue of mixing with chitosan. The further decrease in the intensity of the characteristic peaks of metronidazole in the diffractograms of the prepared hydrogels indicates that the preparation procedure caused a decrement in drug crystallinity although it remained in the crystalline state. The lyophilization process is probably responsible for this decrease in drug crystallinity in the prepared hydrogels as previously explained by Hawe and Frie in their study on the effect of lyophilization on the crystallinity of mannitol hydrate (18).

The results of one-way ANOVA test on the values of formula weight revealed no significant difference between the prepared formulas indicating their weight uniformity. Also, the statistical analysis of data of drug content of the prepared hydrogels proved the uniformity of drug content among the prepared formulas.

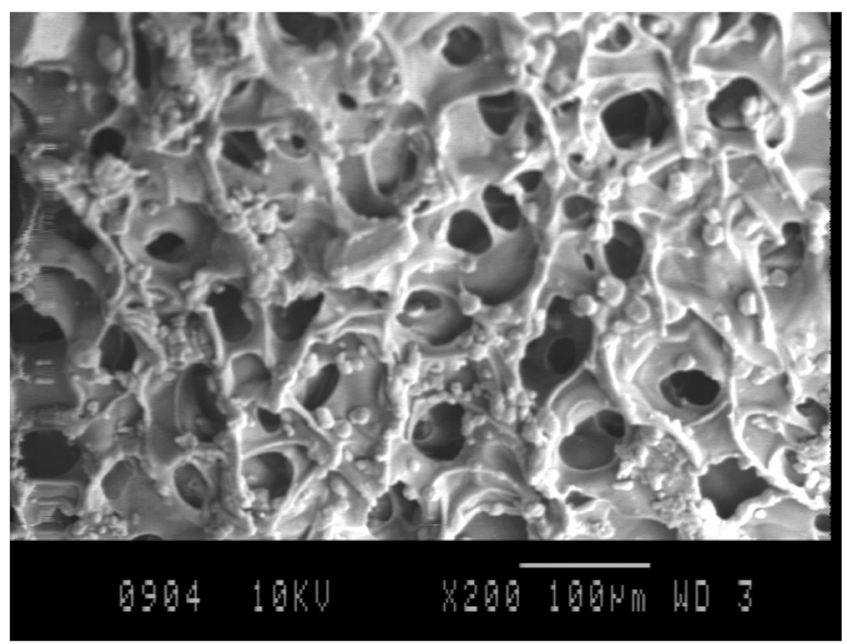

Fig. 6. Scanning electron micrograph of hydrogel formula F1. Composition of $\mathrm{F} 1$ is given in Table I
The drug content of all the prepared formulas met the European Pharmacopoeia's (2002) specifications for singledose preparations as it was in the range of $90-110 \%$ of the theoretical drug content. In addition, one-way ANOVA test revealed that the differences in the results of the residual moisture content of the prepared hydrogel formulas were non-significant. Concerning hydrogel swelling, results reveal that the swelling ability of the prepared hydrogels is highly $\mathrm{pH}$ dependent; as they remarkably swelled in SGF ( $\mathrm{pH} 1.2)$, but their swelling in phosphate buffer of $\mathrm{pH} 7.4$ was comparatively poor. The extensive swelling ability of the prepared hydrogels in SGF ( $\mathrm{pH}$ 1.2) could be attributed to poor ionization of the crosslinking agents (sodium citrate and TPP) at such acidic $\mathrm{pH}$ value. As a result, the electrostatic interaction with chitosan chains was weakened or disappeared. Moreover, protonation of chitosan free ammonium groups at such acidic $\mathrm{pH}$ resulted in their repulsion and favored the swelling of the formulas or even their dissociation. Similarly, the poor swelling ability of the prepared hydrogels in phosphate buffer ( $\mathrm{pH}$ 7.4) could be attributed to the reservation of the electrostatic attraction between the crosslinker ions and chitosan at alkaline $\mathrm{pH}$. However, the slight increase in weight of the hydrogels in this medium might be explained by hydration of the unbound amine groups of chitosan.

The difference in the swelling behavior of formulas prepared using the same chitosan, but different crosslinking agents might indicate that when citrate ions were used they produced higher crosslinking density within the $30 \mathrm{~min}$ allowed for the crosslinking step. This came in accordance with the results of Shu et al. (15) who reported the pH sensitivity of citrate-crosslinked chitosan films and Shu and Zhu (19), who studied the swelling behavior of ionically crosslinked chitosan beads. The high ability of the prepared hydrogel formulas to float over the $24 \mathrm{~h}$ of the experiment can be mainly attributed to the highly porous nature of the formulas probably due to the lyophilization process (20).

The release profiles of metronidazole from the prepared hydrogel formulas clearly signify that the hydrogels exhibited $\mathrm{pH}$-dependent release patterns, where drug release was preferable in SGF ( $\mathrm{pH}$ 1.2) than in phosphate buffer of $\mathrm{pH} 7.4$.

A $2^{3}$ factorial experiment was designed to study the effect of different formulation factors on the drug release from the 

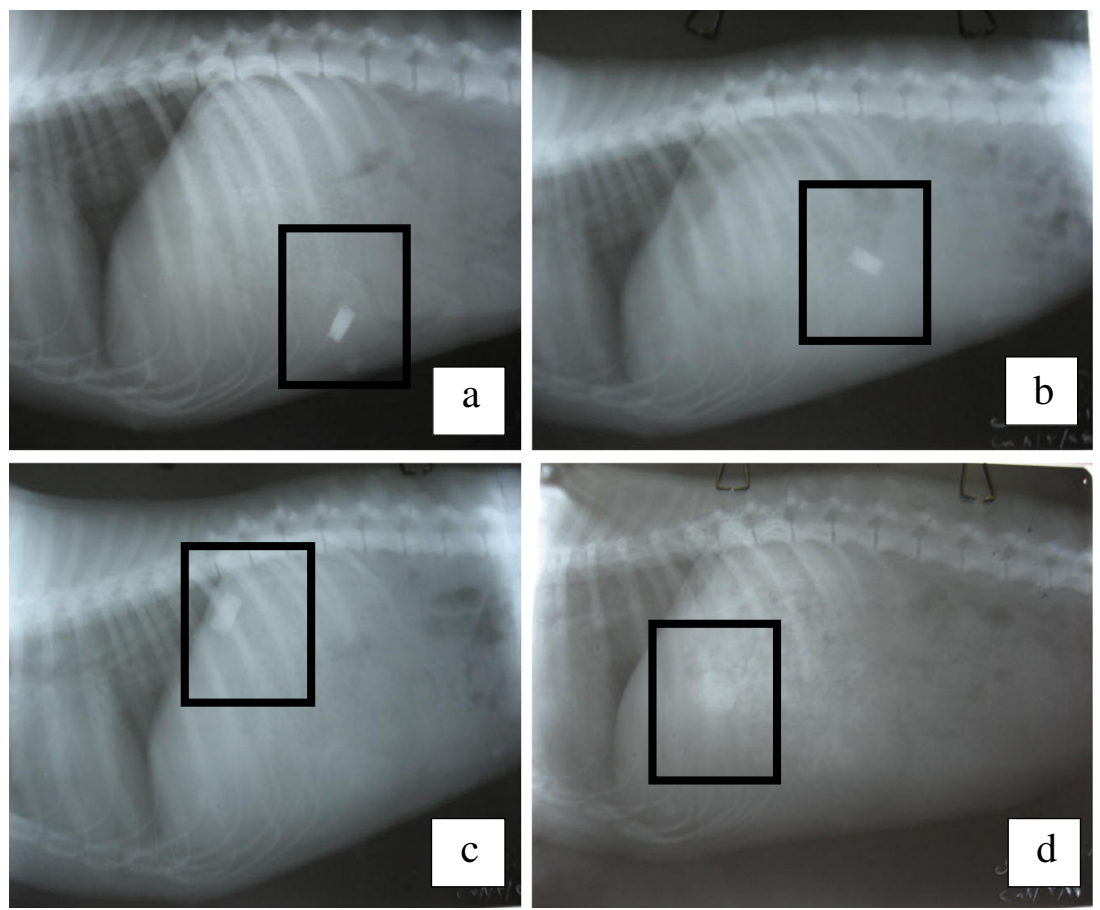

Fig. 7. Radiophotograph of dog's abdomin $4 \mathrm{~h}$ (a), $8 \mathrm{~h}$ (b), $24 \mathrm{~h} \mathrm{(c)}$, and $48 \mathrm{~h}$ (d) after ingestion of the prepared radio-opaque floating chitosan hydrogel formula F1. Composition of $\mathrm{F} 1$ is given in Table I

prepared hydrogels in SGF ( $\mathrm{pH} 1.2)$. The investigated formulation factors were the molecular weight of the used chitosan, the type of the used crosslinking agent as well as its concentration. The $t_{50 \%}$ was chosen to be the parameter for comparison. The effects of formulation factor on $t_{50 \%}$ as well as the interactions between them were evaluated by two-way ANOVA test. Fisher's PLSD was performed to determine the source of difference in ANOVA tables. The statistical analysis of the results of $t_{50 \%}$ showed that all the investigated formulation factors had significant effects on the drug release as revealed from the calculated $p$-values. Concerning the molecular weight of chitosan, using medium molecular weight chitosan in hydrogel preparation (formulas F5-F8) resulted in significant retardation in drug release (mean difference $=264.13, p<0.0001$ ) compared with formulas F1-F4, respectively, where high molecular weight chitosan was used. This might be contributed to the faster diffusion of the crosslinker solution through medium rather than high molecular weight chitosan matrices within the 30 -min period allowed for crosslinking, resulting in higher crosslinking density and consequently slower drug release.

The used crosslinking agent is another formulation factor that was studied for its effect on drug release from the prepared hydrogels. Statistical analysis of results of $t_{50 \%}$ shows that using TPP, as a crosslinking agent, caused significant faster drug release from the prepared hydrogel formulas (F1, F2, F5, and F6) than that from corresponding formulas where sodium citrate was used (F3, F4, F7, and F8; mean difference $=156.38, p<0.0001)$. Due to its larger molecular size, TPP is expected to diffuse into hydrogel matrices much slower than citrate ions do resulting in less-dense crosslinking and as a result, faster drug release. Concerning the third studied formulation factor-the used crosslinker concentration-it significantly affected the values of $t_{50 \%}$ for both TPP and sodium citrate (mean difference $=30.88, p<0.05$ ). The increase in crosslinker concentration in formulations F3 and F5 compared with formulas F4 and F6, respectively, resulted in significant prolongation of $t_{50} \%$. This is logic as higher electrolyte concentration produced higher crosslinking density and consequently, more difficult drug release.

Two-way ANOVA test on results of $t_{50 \%}$ also reveals that there is a significant interaction between the effects of chitosan type and ion type of the crosslinking agent on metronidazole release $(F=817.027, p \leq 0.0001)$. The effect of chitosan type was more pronounced with citrate ions when used as a crosslinking agent than with TPP. On the other hand, using the same crosslinking agent, the effect of chitosan type did not differ significantly by changing the crosslinker concentration.

Also, the interaction between the effect of crosslinking ion type and its concentration was statistically non- significant with $F$ value $=0.742$ and $p$ value $=0.414$. However, the interaction between the effects of the three studied factors (chitosan type, crosslinker ion type, and crosslinker concentration) on the values of $t_{50 \%}$ was significant with $p$ value less than 0.0001 . This may explain the shorter $t_{50 \%}$ obtained from formulas F1 and F7 when compared with formulas F2 and F8, respectively, although the former formulas contain higher concentrations of the corresponding crosslinker.

To determine the kinetic model of metronidazole release from the prepared hydrogels, the release results of the drug from each of the prepared formulas were subjected to kinetic studies applying zero-order, first-order, and Higushi diffusion equations. The preference of a certain release model was based on the calculated regression coefficient $\left(R^{2}\right)$ of the best 

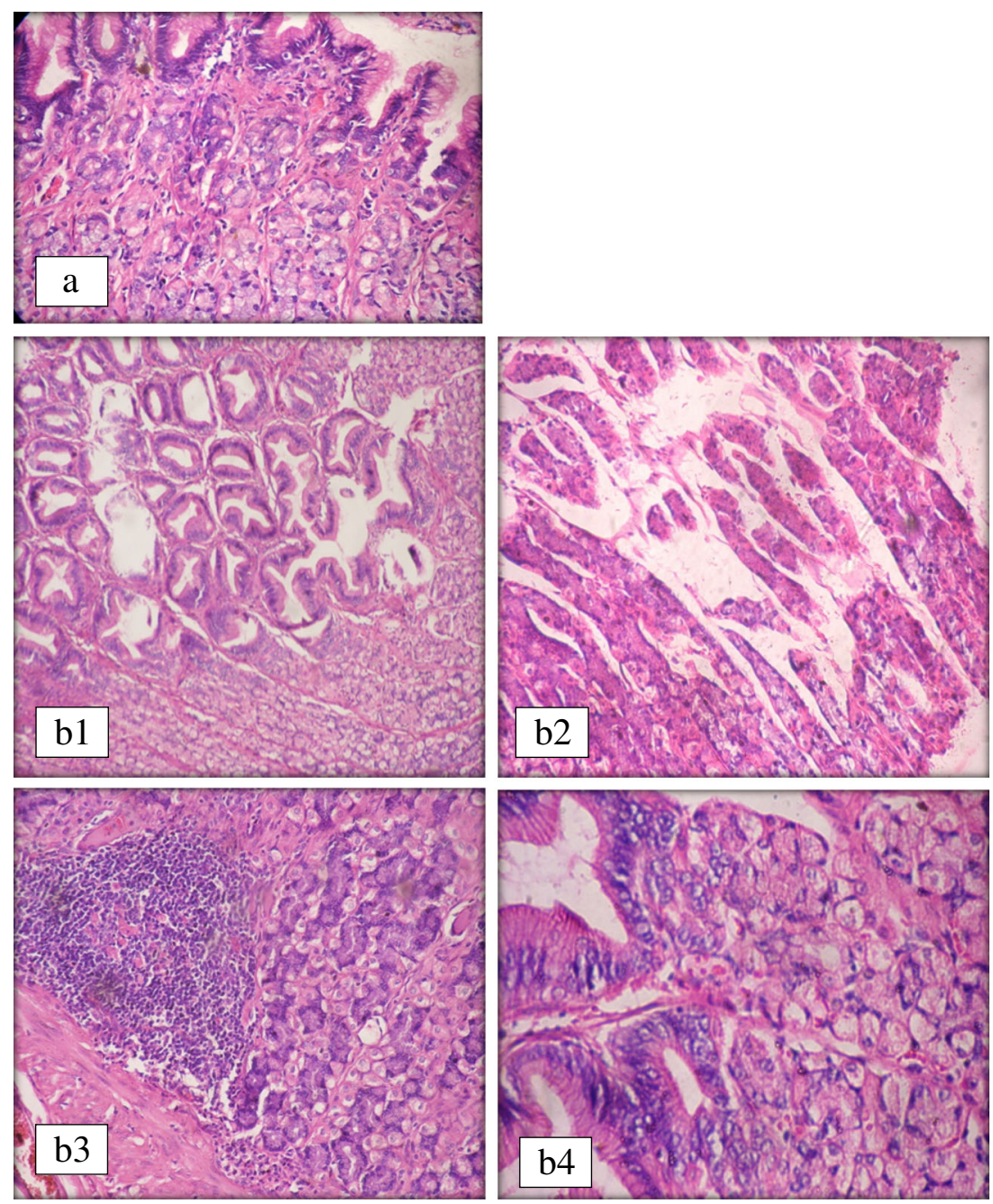

Fig. 8. Histological examination of the gastric mucosa of control dogs participated in the in vivo studies: negative control $\operatorname{dog}(a)$, positive control dogs (group A animals) (b1-b4) Magnifications, $a, b 2, b 3 \times 200, b 1 \times 100$, and $b 4 \times 400$

statistical line of each equation. Previous researchers who studied the release kinetics of drugs from hydrogel matrices reported that the release followed Higuchi diffusion kinetics in the majority of cases (21). However, in our study the large dose of metronidazole as well as the rapid swelling of the prepared hydrogels could be responsible for the first-order kinetics that was followed by five out of the eight prepared hydrogel formulas. Since some values of $\left(R^{2}\right)$ were very close, differ only by the second or third digit, the release results were re-analyzed according to the empirical formula of Ritger and Peppas (17). The calculated values of $(n)$ in such empirical formula did not exceed unity for any of the prepared formulas indicating non-Fickian diffusion, with both diffusion and relaxation controlled systems.

The above discussion of the results reveals the superiority of formula F1 to other prepared hydrogel formulas. Formula F1 swelled significantly $(700 \%)$ within the first $4 \mathrm{~h}$ and retained its configuration without dissociation for $24 \mathrm{~h}$. This intact gel structure is favorable because of the anti-ulcer properties of chitosan discussed by previous researchers $(11,12)$. In addition, Formula F1 released $65 \%$ of the drug within the first
$6 \mathrm{~h}$ and kept a considerable concentration of the drug over $24 \mathrm{~h}$. For these reasons, formula F1, prepared using $2 \%$ high molecular weight chitosan crosslinked with $2 \%$ TPP solution was chosen for further investigations.

The formula of choice (formula F1) was examined under electron microscope where it showed a highly porous structure. This porous nature was most probably produced by the lyophilization process, and it could be responsible for offering a large surface area and, therefore, a better matrix-solvent interaction allowing faster solvent uptake by the formula. The prepared hydrogel formula F1 was stored for 6 months at room temperature under highly humid conditions (relative humidity $75 \%$ ). The statistical analysis of results of moisture uptake on storage (using one-way ANOVA test) reveals the non- significant effect of humid conditions on the moisture uptake of the formula ( $p$ values $>0.05$ ) even after 6-month storage.

For in vivo evaluation of the selected hydrogel formula F1, dogs were chosen as the animal model for estimation of the efficiency of the prepared formula in eradication of $H$. pylori as they were used by many researchers as an animal 

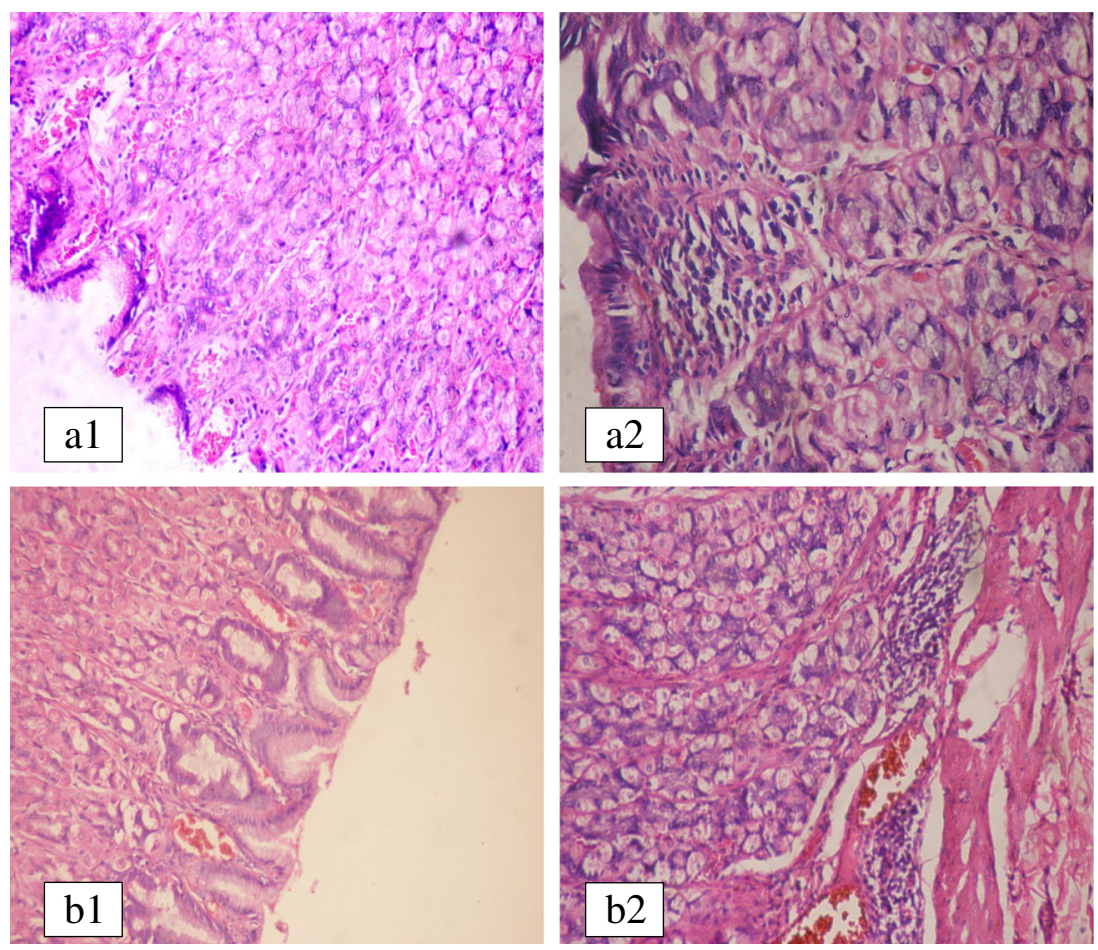

Fig. 9. Histological examination of the gastric mucosa of treated dogs participated in the in vivo studies: group B animals (treated with Flagyl ${ }^{\circledR}$ tablets) $(a 1, a 2)$ and group $\mathrm{C}$ animals (treated with the hydrogel formula F1) $(b 1, b 2)$. Magnifications, $a 1 \times 100, a 2 \times 400$, and $b 1$, $b 2 \times 200$. Composition of F1 is given in Table I

model to study the pathogenesis of such micro-organisms (16). In addition, dogs have been used as the animal model of choice for the evaluation of the performance of different gastro-retentive dosage forms, due to the similarity in the anatomic structure of their stomach and in gastric emptying pattern with human. However, the smaller diameter of the dog's pylorus in comparison with human one may cause extended gastric retention time as explained by Dressman (22).

Visualized by radiographic examination, the swallowed formula F1 existed in the dog's stomach for $48 \mathrm{~h}$. This proves that the hydrogel was not swapped by the housekeeper wave (the phase during which large non-disintegrating particles or dosage forms are emptied from the stomach to the small intestine). In addition, the hydrogel was not emptied by the effect of the migrating mylo-electric cycle of the gastric emptying cycle which occurs approximately every $2 \mathrm{~h}$ in humans and every $1 \mathrm{~h}$ in dogs (23). The radiographs also reveal that the hydrogel had altered its position and turned around during its presence in the stomach as evidence that it did not adhere to the gastric mucosa, instead it floats on the gastric fluids.

Depending on the results of the in vitro release experiment (showing a drug release of about $76 \%$ of the loaded drug in hydrogel formula F1 within $24 \mathrm{~h}$ ), and the radiographic examination of formula F1 (proving its floating and gastro-retentive nature), it can be concluded that the hydrogel formula F1 could be therapeutically beneficial by allowing longer localization of the released metronidazole at the site of its action (stomach).

The gastric biopsies taken from the stomach of the negative- control dog gave negative urease test indicating that the stomachs of the dogs participated in this study were free from Helicobacter species before inoculation of $H$. pylori. Successful colonization of $H$. pylori in the stomach of dogs was proved by positive urease test for gastric biopsies taken from the positive control dogs (group A). Histological examination of group A dogs' stomach—shown in Fig. 8-revealed the presence of the characteristic signs of inflammation.

For the treated animals, histopathological examination of the stomach showed less inflammation in the biopsies taken from animals treated with the prepared hydrogel formula F1 (group C dogs) compared with those treated with Flagyl ${ }^{\circledR}$ tablets (group B ones).

Statistical analysis of data revealed that both Flagyl ${ }^{\circledR}$ and the selected hydrogel formula $\mathrm{F} 1$ produced significant reduction in colony count compared with the untreated animals. Comparing the two treatments, the colony count of dogs treated with the selected hydrogel formula F1 was significantly less than that for animals treated with Flagyl®. This indicates that the selected hydrogel formula F1 was more effective than Flagyl ${ }^{\circledR}$ tablets against $H$. pylori, a result that could be attributed to the gastric retention of the prepared hydrogel in the stomach of dogs, as proved by the X-ray radiography. The gastric- retentive hydrogel released the drug in stomach in a controlled manner, as previously revealed by the in vitro release study. This increased the exposure of bacteria to the drug compared with conventional tablets leading to the conclusion that the prepared hydrogel formula is more effective in eradication of $H$. pylori than the conventional oral metronidazole tablets.

\section{CONCLUSION}

In conclusion, a promising floating metronidazole- containing hydrogel can be prepared using $2 \%$ high molecular weight chitosan as the polymer and $2 \%$ TPP solution as a crosslinker. The prepared hydrogel has the feasibility to be 
retained in the stomach and to release metronidazole in a controlled manner over a period of $24 \mathrm{~h}$. Furthermore, the prepared hydrogel was more effective than oral metronidazole (Flagyl ${ }^{\circledR}$ tablets) in the eradication of $H$. pylori in infected dog's stomach. Keeping in mind that metronidazole in the prepared formula was used at a lower dose level (250 mg, once daily) than in the conventionally used oral triple therapy (500 $\mathrm{mg}$, three times daily), the results of this paper indicated that the prepared hydrogel could be considered as a promising site-specific delivery system for the treatment of peptic ulcer caused by $H$. pylori. For further improvement of the efficiency of the prepared formula, the treatment duration can be elongated, accompanied by the incorporation of another antibiotic along with a proton pump inhibitor in the prepared hydrogel.

\section{ACKNOWLEDGMENTS}

The authors would like to acknowledge The National Hepatology and Tropical Medicine Research Institute, Cairo, Egypt, for providing gastric biopsies from patients with gastritis and gastric and duodenal ulcers. The authors also thank The Faculty of Veterinary Medicine Cairo University, for providing the facilities during the in vivo study.

\section{REFERENCES}

1. Marshall BJ, Warren JR. Unidentified curved bacilli in the stomach of patients with gastritis and peptic ulceration. Lancet. 1984;1:1311-5.

2. Wolle K, Malfertheiner P. Treatment of Helicobacter pylori. Best Pract Res Clin Gastroenterol. 2007;21:315-24.

3. Sweetman SC. Martindale; the complete drug reference. 33rd ed. London: The Pharmaceutical Press; 2002. p. 759-67.

4. Bardonnet PL, Faivre V, Pugh WJ, Piffaretti JC, Falson F. Gastroretentive dosage forms: overview and special case of Helicobacter pylori. J Control Release. 2006;111:1-18. doi:10.1016/j.jconrel.2005.10.031.

5. Desai S, Bolton S. A floating controlled release drug delivery system: in vitro-in vivo evaluation. Pharm Res. 1993;10:1321-5.

6. Peppas NA. Hydrogels and drug delivery. Curr Opin Colloid Interface Sci. 1997;2:531-7. doi:10.1016/S1359-0294(97)80103-3.

7. Ishak RAH, Awad GAS, Mortada ND, Nour SAK. Preparation, in-vitro and in-vivo evaluation of stomach-specific metronidazole loaded alginate beads as local anti-Helicobacter pylori therapy. J Control Release. 2007;119:207-14.
8. Qiu Y, Park K. Environment-sensitive hydrogels for drug delivery. Adv Drug Deliv Rev. 2001;53:321-39.

9. Rajinikanth PS, Balasubramaniam J, Mishra B. Development and evaluation of a novel floating in situ gelling system of amoxicillin for eradication of Helicobacter pylori. Int J Pharm. 2007;335:114-22.

10. Boriwanwattanarak P, Ingkaninan K, Khorana N, Viyoch J. Development of curcuminoids hydrogel patch using chitosan from various sources as controlled-release matrix. Int J Cosmet Sci. 2008;30:205-18. doi:10.1111/j.1468-2494.2008.00437.x.

11. Ueno H, Mori T, Fujinaga T. Topical formulations and wound healing applications of chitosan. Adv Drug Deliv Rev. 2001;52:105-15.

12. Burkatovskaya M, Castano AP, Demidova-Rice TN, Tegos GP, Hamblin MR. Effect of chitosan acetate bandage on wound healing in infected and noninfected wounds in mice. Wound Repair Regen. 2008;16:425-31. doi:10.1111/j.1524475X.2008.00382.x.

13. Malaekeh-Nikouei B, Sajadi Tabassi SA, Jaafari MR. Preparation, characterization, and mucoadhesive properties of chitosancoated microspheres encapsulated with cyclosporine A. Drug Dev Ind Pharm. 2008;34:492-8. doi:10.1080/03639040701744004.

14. Bergera J, Reista M, Mayera JM, Feltb O, Peppasc NA, Gurnyb R. Structure and interactions in covalently and ionically crosslinked chitosan hydrogels for biomedical applications. Eur J Pharm Biopharm. 2004;57:19-34.

15. Shu XZ, Zhu KJ, Song W. Novel pH-sensitive citrate cross-linked chitosan film for controlled drug release. Int J Pharm. 2001;212:19-28.

16. Rossi G, Rossi M, Vitali CG, Fortuna D, Burroni D, Pancotto L, et al. A conventional beagle dog model for acute and chronic infection with Helicobacter pylori. Infect Immun. 1999;67:311220.

17. Ritger PL, Peppas NA. A simple equation for description of solute release. II. Fickian and anomalous release from swellable devices. J Control Release. 1987;5:37-42. doi:10.1016/01683659(87)90035-6.

18. Hawe A, Frie W. Impact of freezing procedure and annealing on the physico-chemical properties and the formation of mannitol hydrate in mannitol-sucrose- $\mathrm{NaCl}$ formulations. Eur J Pharm Biopharm. 2006;64:316-25.

19. Shu XZ, Zhu KJ. Controlled drug release properties of ionically cross-linked chitosan beads: the influence of anion structure. Int J Pharm. 2002;233:217-25. doi:10.4103/0250-474X.40326.

20. Jiankang H, Dichen L, Yaxiong L, Bo Y, Bingheng L, Qin L. Fabrication and characterization of chitosan/gelatin porous scaffolds with predefined internal microstructures. Polymer. 2007:48:4578-88. doi:10.1007/s13726-012-0019-0.

21. Agnihotri SA, Aminabhavi TM. Novel interpenetrating network chitosan-poly(ethylene oxide-g-acrylamide) hydrogel microspheres for the controlled release of capecitabine. Int J Pharm. 2006;324:103-15. doi:10.1016/ j.ijpharm.2006.05.061.

22. Dressman JB. Comparison of canine and human gastrointestinal physiology. Pharm Res. 1986;3:123-31. doi:10.1023/ A:1016353705970.

23. Arora S, Ali J, Ahuja A, Khar RK, Baboota S. Floating drug delivery systems: a review. AAPS Pharm Sci Tech. 2005;6:372-90. doi:10.1208/pt070117. 